\title{
Tables
}

Table 1- Comparison of dissolved $\mathrm{Cd}, \mathrm{Cu}, \mathrm{Pb}$ and $\mathrm{Ag}$ analyses obtained in the present study by the ID-ICPMS method and of dissolved Mn obtained by the internal standard addition and ICPMS detection, with the consensus values reported by the SAFe program (in the surface-S and deep-D2 samples) and in the North Atlantic GEOTRACES (in the surface-GS and deep-GD samples) Reference Samples (updated in November 2011). All \pm terms represent standard deviation from average values.

\begin{tabular}{|c|c|c|c|c|c|c|c|c|}
\hline & \multicolumn{2}{|c|}{ SAFe concentration } & \multicolumn{2}{|c|}{ Consensus value SAFe } & \multicolumn{2}{|c|}{ GEOTRACES concentration } & \multicolumn{2}{|c|}{ Consensus value GEOTRACES } \\
\hline & $S$ & D2 & $S$ & D2 & GS & GD & GS & GD \\
\hline $\mathrm{Cd}$ & $\begin{array}{c}0.77 \pm 0.96 \mathrm{pM} \\
(n=4)\end{array}$ & $\begin{array}{c}1034 \pm 15 \mathrm{pM} \\
(n=8)\end{array}$ & $1.00 \pm 0.2 \mathrm{pM}$ & $986 \pm 27 \mathrm{pM}$ & $\begin{array}{c}2.86 \pm 0.37 \mathrm{pM} \\
(n=3)\end{array}$ & $\begin{array}{c}287 \pm 7 \mathrm{pM} \\
(n=4)\end{array}$ & $2.4 \pm 0.4 \mathrm{pM}$ & $273 \pm 6 \mathrm{pM}$ \\
\hline $\mathrm{Cu}$ & $\begin{array}{c}0.56 \pm 0.03 \mathrm{nM} \\
(n=10)\end{array}$ & $\begin{array}{c}1.39 \pm 0.13 \\
\mathrm{nM}^{\mathrm{a}} \\
(n=9)\end{array}$ & $0.51 \pm 0.05 \mathrm{nM}$ & $2.25 \pm 0.11 \mathrm{nM}$ & $\begin{array}{c}0.72 \pm 0.07 \\
\mathrm{nM}^{\mathrm{a}} \\
(n=4)\end{array}$ & $\begin{array}{c}1.12 \pm 0.04 \\
\mathrm{nM}^{\mathrm{a}} \\
(n=4)\end{array}$ & $0.83 \pm 0.08 \mathrm{nM}$ & $1.55 \pm 0.13 \mathrm{nM}$ \\
\hline Mn & $\begin{array}{c}0.93 \pm 0.06 \mathrm{nM} \\
(n=4)\end{array}$ & $\begin{array}{c}0.49 \pm 0.07 \mathrm{nM} \\
(n=4)\end{array}$ & $\begin{array}{c}0.79 \pm 0.06 \\
\mathrm{nM}^{\mathrm{b}}\end{array}$ & $\begin{array}{c}0.35 \pm 0.06 \\
\mathrm{nM}^{\mathrm{b}}\end{array}$ & Not measured & Not measured & $1.45 \pm 0.17 \mathrm{nM}$ & $0.21 \pm 0.04 \mathrm{nM}$ \\
\hline $\mathrm{Pb}$ & $\begin{array}{c}51.2 \pm 2.2 \mathrm{pM} \\
(n=11)\end{array}$ & $\begin{array}{c}36.3 \pm 1.4 \mathrm{pM} \\
(n=7)\end{array}$ & $47.6 \pm 2.4 \mathrm{pM}$ & $27.7 \pm 1.8 \mathrm{pM}$ & $\begin{array}{c}31.1 \pm 1.6 \mathrm{pM} \\
(n=4)\end{array}$ & $\begin{array}{c}43.8 \pm 3.2 \mathrm{pM} \\
(n=4)\end{array}$ & $29.5 \pm 2.1 \mathrm{pM}$ & $42.2 \pm 1.3 \mathrm{pM}$ \\
\hline $\mathrm{Ag}$ & $\begin{array}{c}3.5 \pm 0.7 \mathrm{pM} \\
\quad(n=3)\end{array}$ & $\begin{array}{c}26.2 \pm 0.9 \mathrm{pM} \\
(n=5)\end{array}$ & No value & No value & $\begin{array}{c}2.7 \pm 0.9 \mathrm{pM} \\
\quad(n=3)\end{array}$ & $\begin{array}{c}13.1 \pm 1.0 \mathrm{pM} \\
(n=4)\end{array}$ & No value & No value \\
\hline
\end{tabular}

a The UV oxidation of the samples might be required to obtain an accurate value for dissolved Cu using this method (Milne et al., 2010) ; ${ }^{\mathrm{b}}$ In general methods based upon ICP-MS yield higher dissolved Mn concentrations than methods based upon catalytic-enhanced flow injection. Furthermore there are significant differences between UV treatment and non-

UV treated samples for dissolved Mn (http://www.geotraces.org/images/stories/documents/intercalibration/Files/Reference_Samples_November11/SAFe_Ref_Mn.pdf). 
Figures

Figure 1

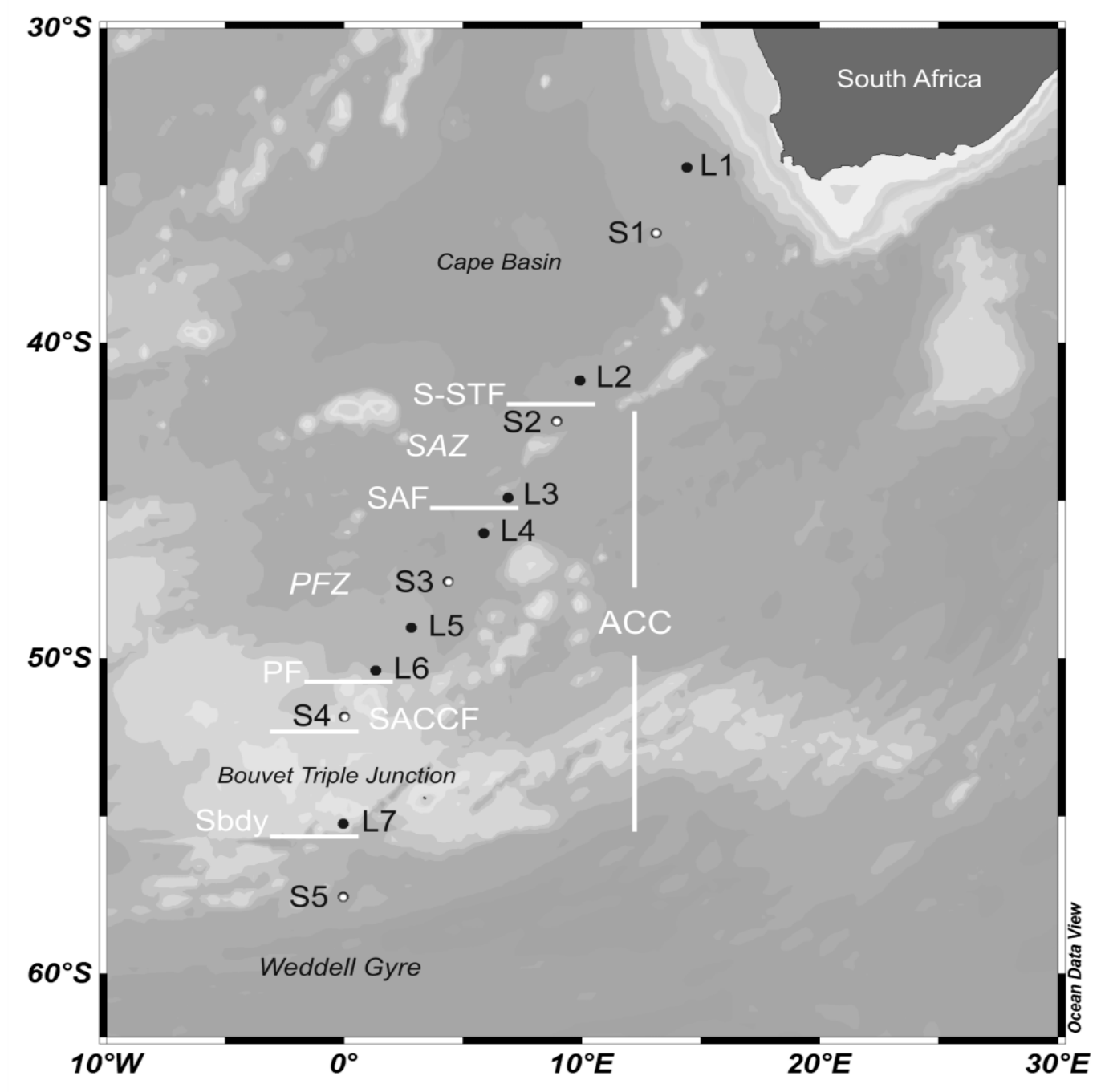


Figure 2
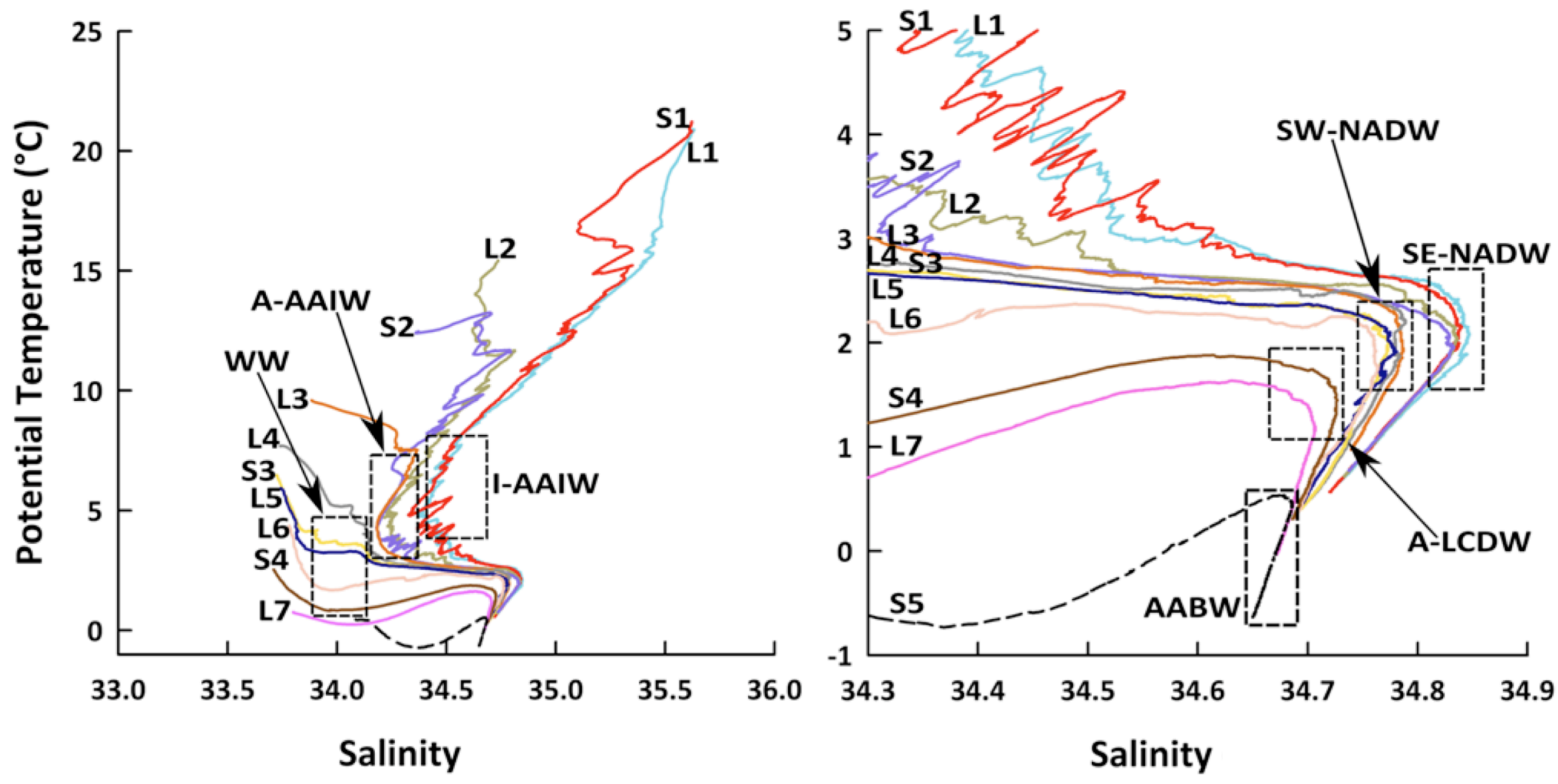


\section{Figure 3}
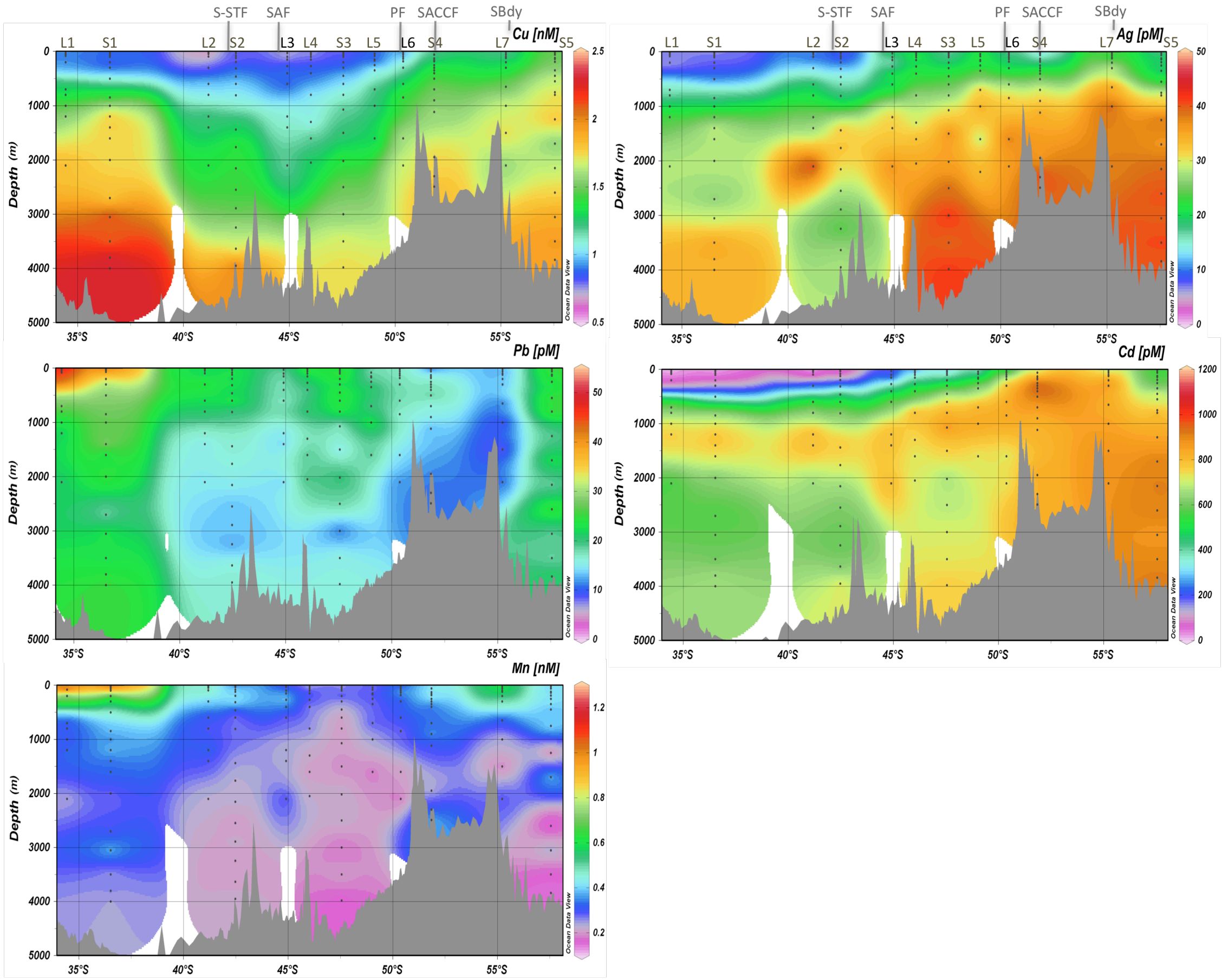

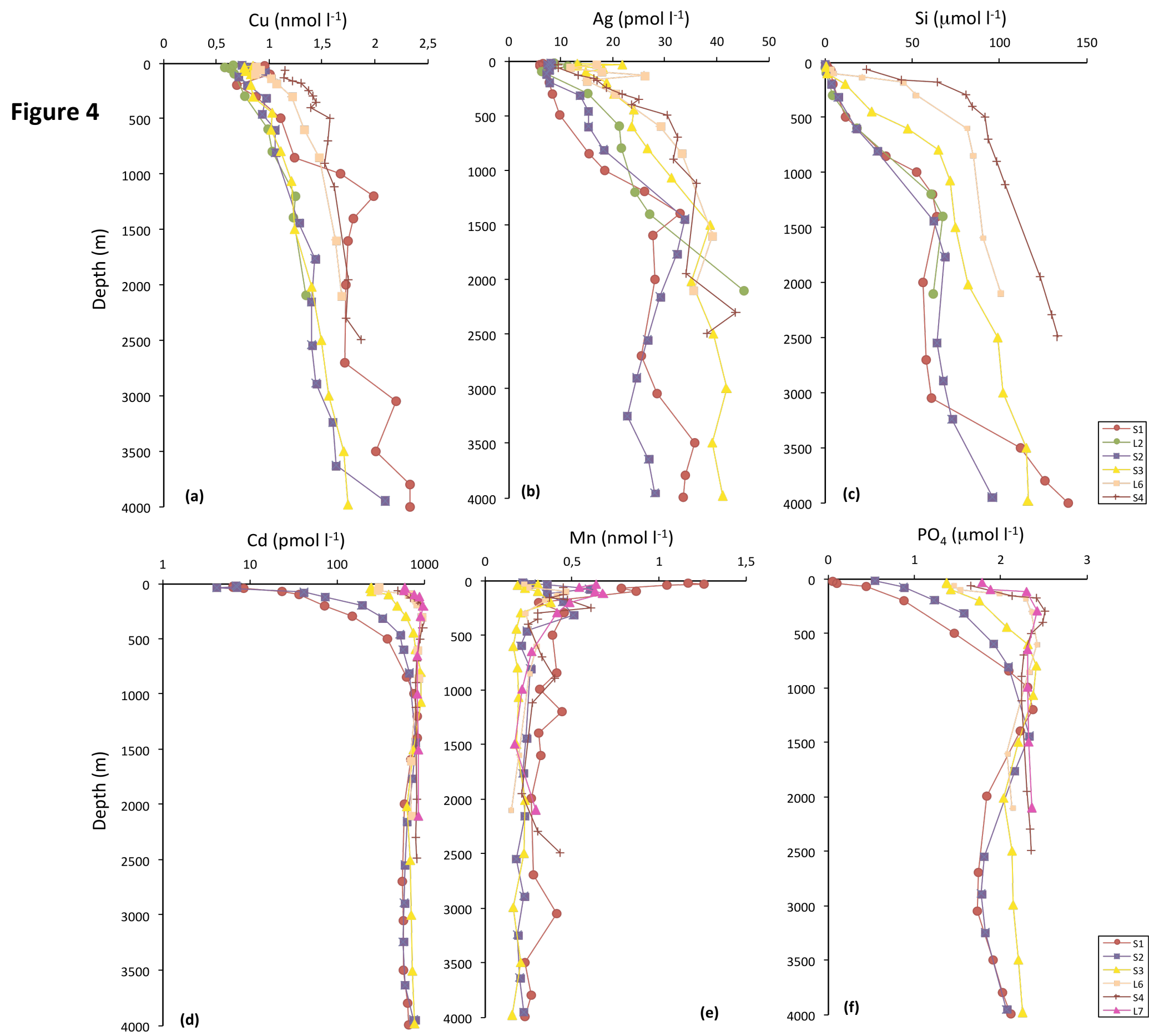
Figure 5
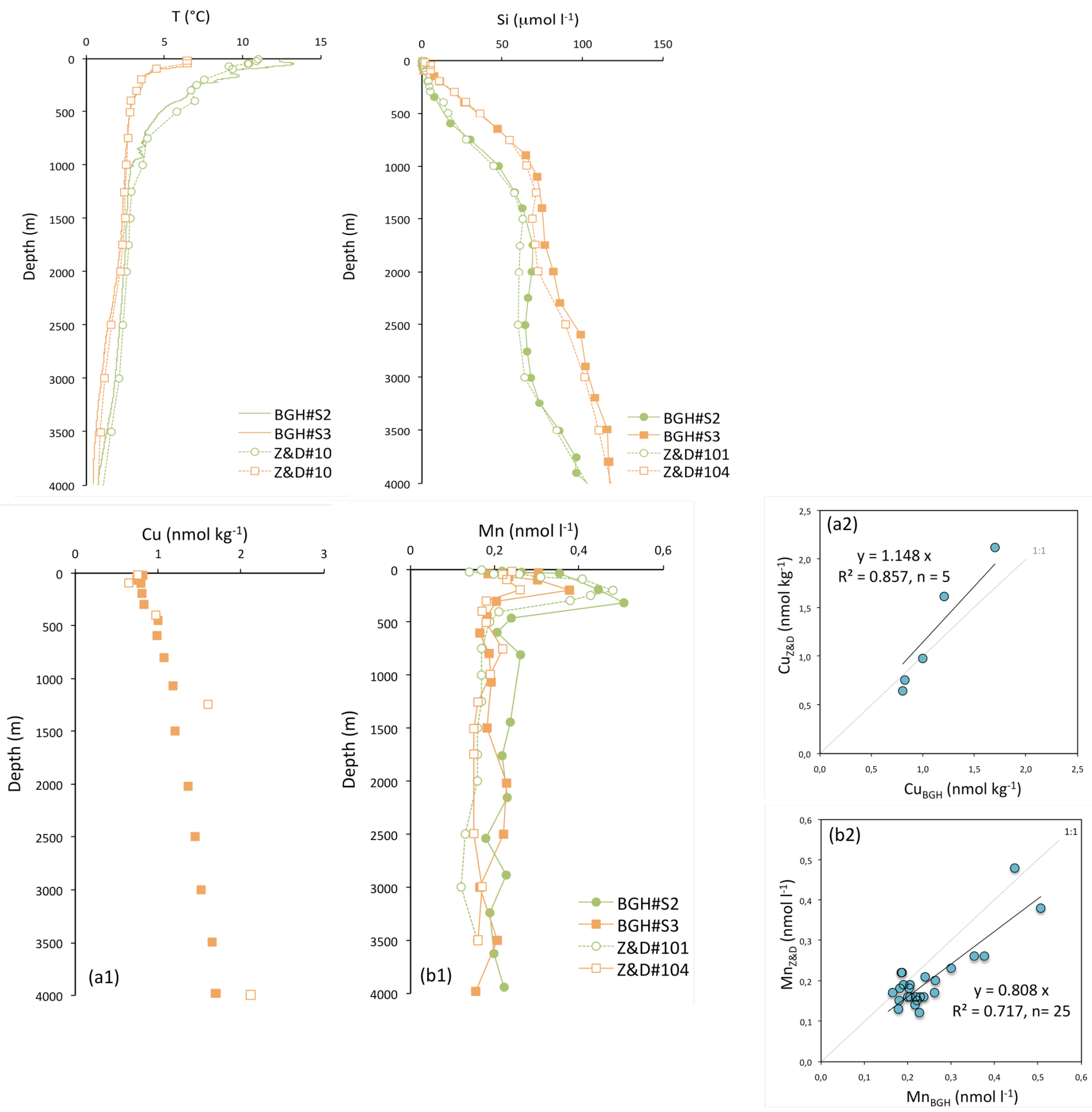
Figure 6

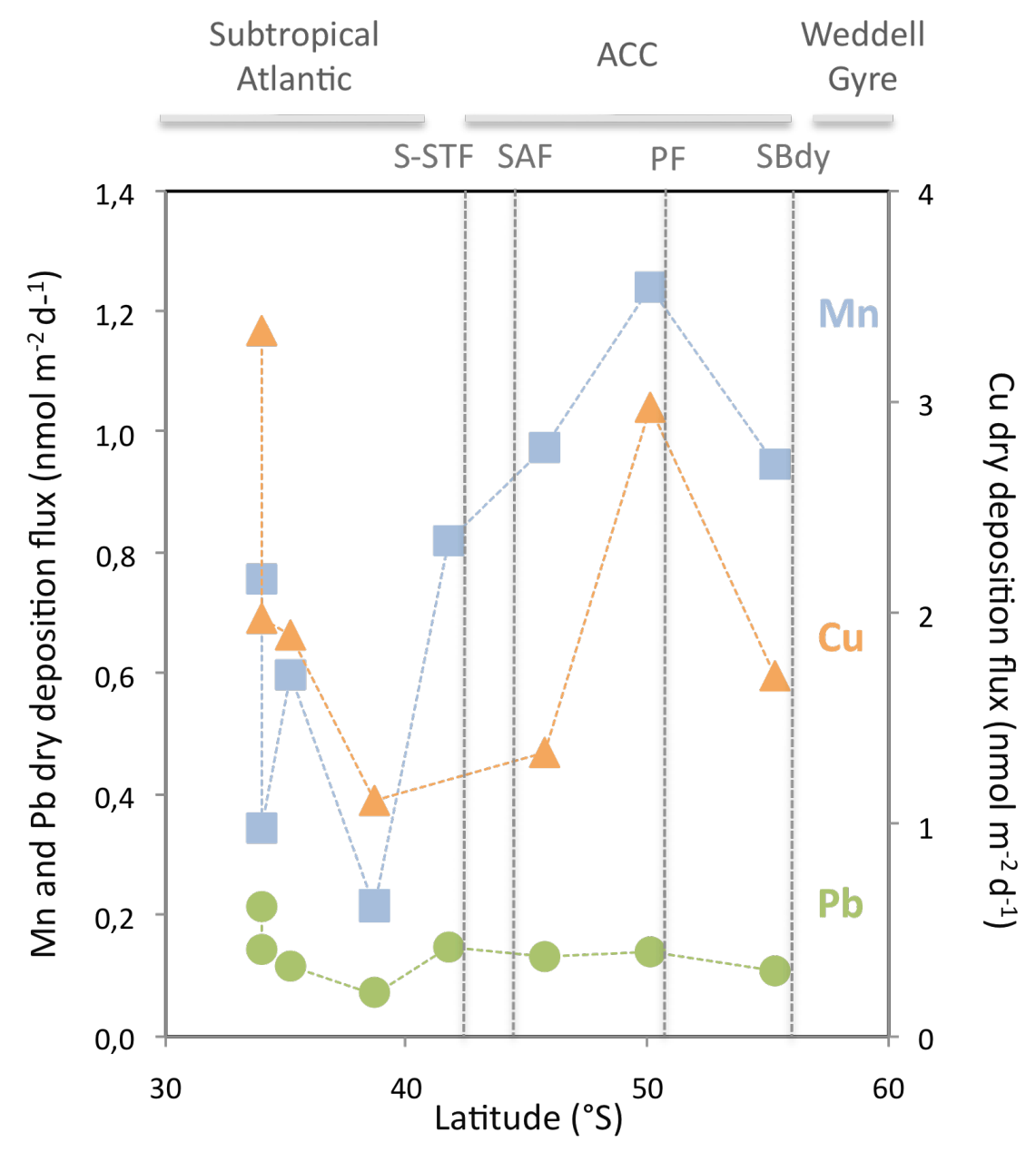


Figure 7
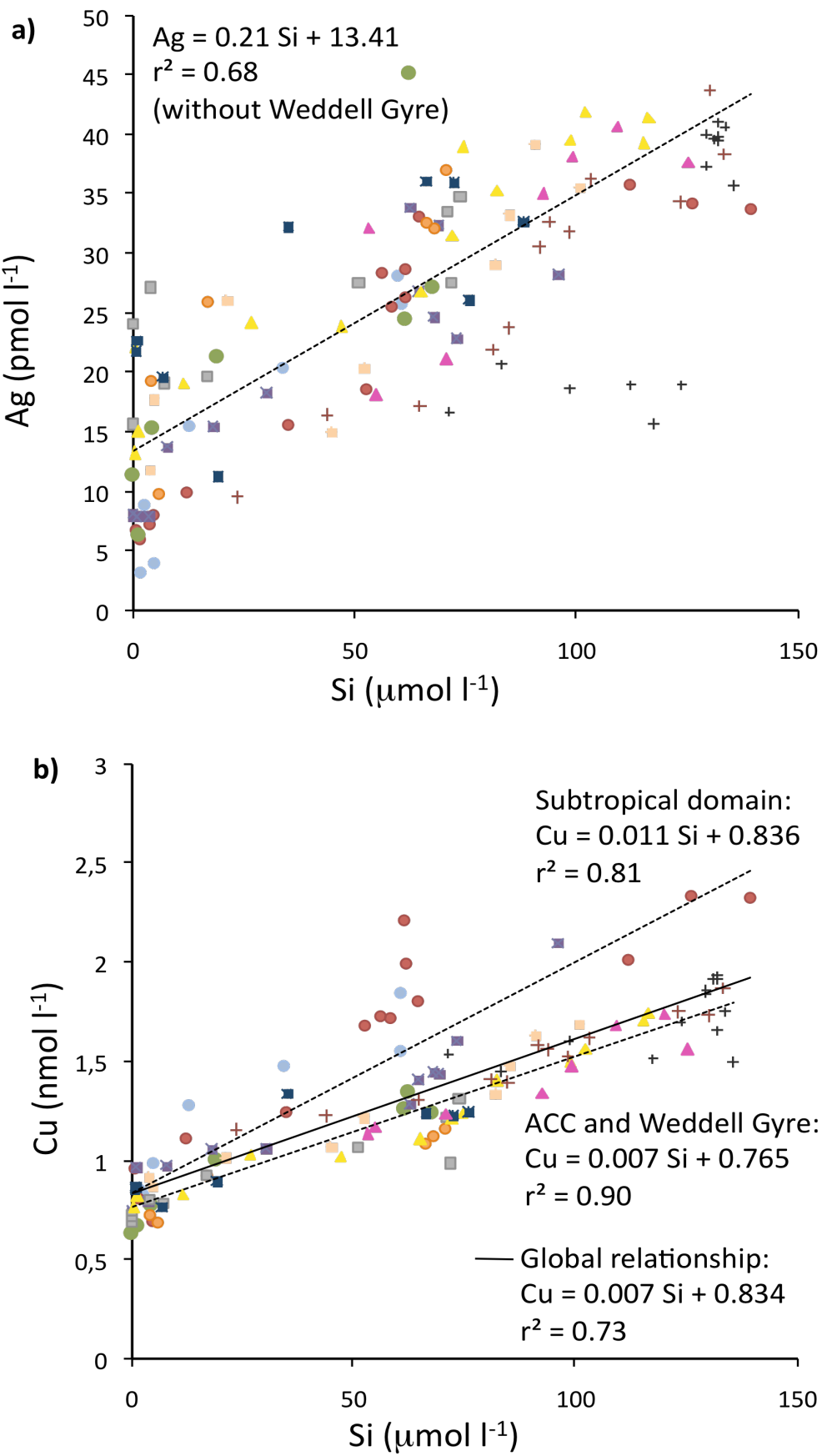
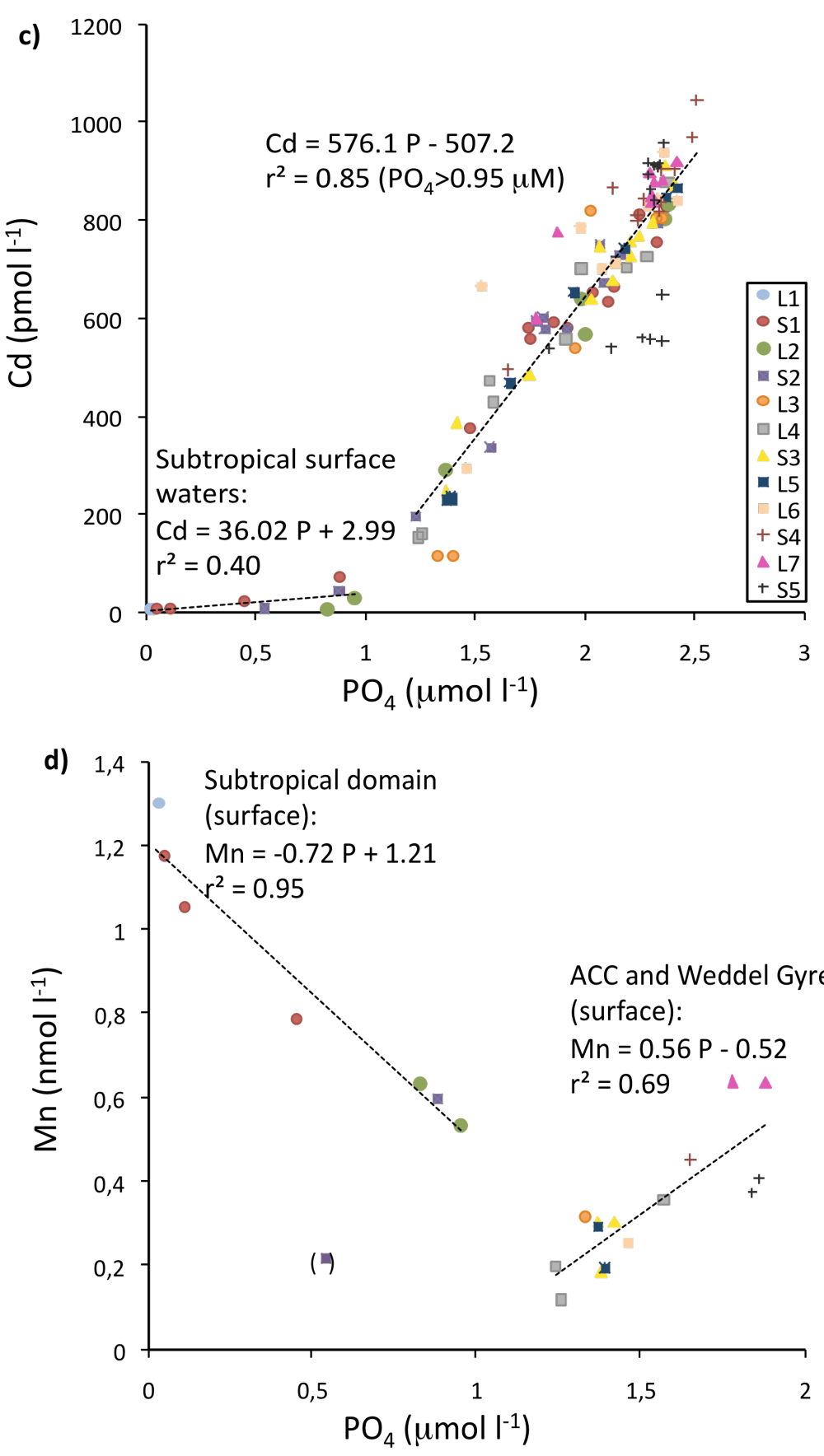
Figure 8

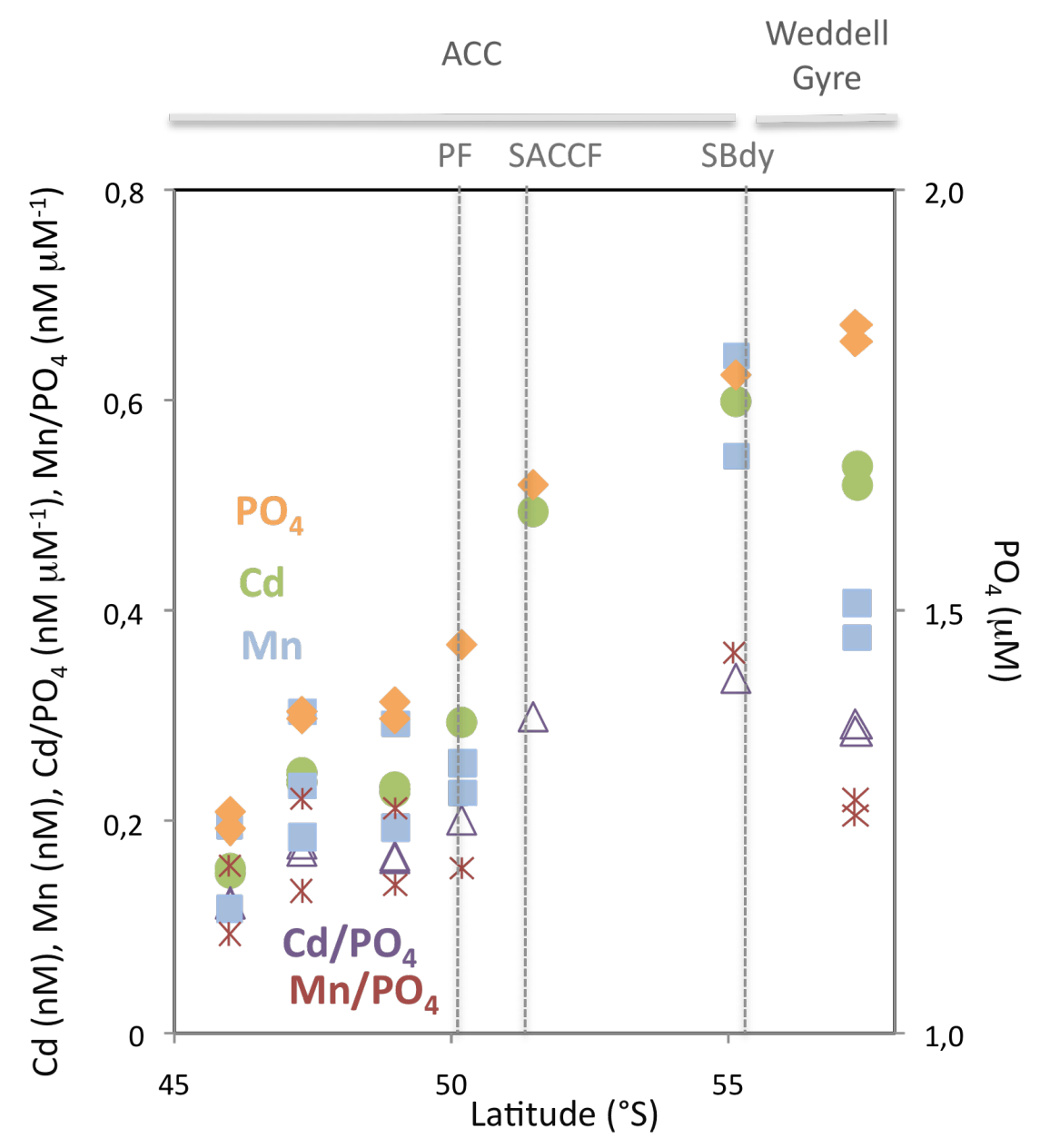

